\title{
Proposal for Distribution of a Low-Phase-Noise Oscillator Signal in Forthcoming Fifth-Generation Mobile Network by Rradio-Over-Fibre Technology
}

\author{
Mehmet Alp Ilgaz, Bostjan Batagelj \\ University of Ljubljana, Faculty of Electrical Engineering, ICT Department, Radiation and Optics Laboratory \\ Trzaska cesta 25, 1000 Ljubljana, Slovenia \\ mehmet.ilgaz@fe.uni-lj.si
}

\begin{abstract}
This article presents a solution that needs only one low-phase-noise oscillator for many cellular network base-stations. The proposed approach can provide a solution that is especially suitable for the forthcoming fifth-generation (5G) high-capacity radio system based on millimetre-wave $(\mathrm{mmW})$ frequency bands, where the phase noise is one of main limiting parameters. The stable and low-phase-noise signal, which can be generated by an advanced opto-electronic oscillator (OEO), is distributed to remote antenna units over a passive optical network infrastructure by radio-over-fibre (RoF) technology. Besides the cost effectiveness, this solution can decrease the size and complexity of base-stations, the number of which will increase in 5G cellular networks due to a reduction in the cell size. This article also presents the key building block for such a RoF system, which is a high-stability OEO.
\end{abstract}

Keywords - Radio Over Fibre; Opto-Electronic Oscillator; Low Phase Noise; Millimetre-Wave; 5G Mobile Network

\section{INTRODUCTION}

The forthcoming fifth-generation $(5 \mathrm{G})$ mobile network is expected to handle a very large amount of data traffic with sub-ms latency [1]. For such an efficient broadband mobile network, frequencies in the millimetre-wave $(\mathrm{mmW}) \mathrm{range}(30-300 \mathrm{GHz}) \mathrm{need}$ to be used [2]. Since the atmospheric scattering and attenuation of mmWs is high, signal propagation is limited to a small area and the cell size needs to be reduced to a picocell $(100 \mathrm{~m})$ or even a femtocell $(10 \mathrm{~m})$. In contrast to the upgrade from the $3 \mathrm{G}$ to the $4 \mathrm{G}$ mobile network, where the number of cells did not increase dramatically [3], it is expected that the upgrade to the 5G mobile network will increase the number of cell base-stations. Smaller base cells can be applied even with today's technology, but at the same time mobile operators are anticipating that the complexity, energy consumption and cost of the base-stations will decrease. As we can see, one of the possibilities presented in this paper is to lower the system costs while increasing its performance by introducing distributed network elements.

Different modulation schemes and multiplexing techniques are proposed for spectra saving and increasing the wireless system's capacity [4], [5]. In all these cases it is clear that the phase noise of the transmitted signal is playing an important role [6]. Today's mobile-communications transceivers employ oscillators that provide the periodic signal needed for frequency translation in the transceiver circuits and for the timing of the digital circuits. When radio interfaces move to the mmW region [7] a low phase noise is even more difficult to achieve [8] since the phase-noise floor is increasing when the oscillators are operating at high frequencies. In our opinion a low-phase-noise frequency oscillator in the range of mmWs is critical for $5 \mathrm{G}$ and is a key technology enabler.

This paper presents a technical solution for the distribution of a low-phase-noise oscillator signal in the forthcoming $5 \mathrm{G}$ mobile networks. First, the benefits of radio-over-fibre technology are described. In the third section the opto-electronic oscillator (OEO) is presented as the main device in the proposed system solution because of its low phase noise. Besides a microwave output it has an optical output that can be used to distribute the oscillator signal to the base-stations, as described in the fourth section, which is followed by the conclusion in the fifth section.

\section{BASICS OF RADIO OVER FIBRE TECHNOLOGY}

It is well known that a fibre-optic communication link is superior to a wireless radio connection in terms of both the main important parameters that determine the value of telecommunication lines [9]. The first is the range of the telecommunication line measured in units of length, where optical fibre is the absolute winner with an attenuation of $0.2 \mathrm{~dB}$ per kilometre, in comparison to the wireless radio link, where the signal attenuation is higher and increases with frequency. The second parameter is the link capacity, which measures the amount of information transmitted in a time frame, where the optical fibre's broadband properties make it a practically bandwidth-unlimited medium with a slow approach to the "fibre-wall" [10].

The basic idea of radio-over-fibre (RoF) technology, which uses the transmission of radio frequency (RF) signals over fibreoptic links, is to take advantage of fibre's low attenuation and high bandwidth [11]. Since a fibre-optic line has a large bandwidth, it allows the transmission of RF, microwave or even $\mathrm{mmW}$ signals. There are several principles behind the various RF signal transport methods in RoF technology [11]. The simplest method for optically distributing a RF signal is simply to directly modulate the intensity of the laser-diode (LD) source with the RF signal and to use direct detection (DD) at the photodiode (PD) to receive 
the RF signal. For the transmission of higher-frequency signals such as mmWs the intensity modulation (IM) is not possible by directly modulating the LD's current due to the LD's limited bandwidth. Fortunately, there is another option for intensity modulation where the LD operates in continuous-wave $(\mathrm{CW})$ mode and then an external modulator, such as electro-absorption modulator or Mach-Zehnder Modulator (MZM), is used to modulate the intensity of the light [12]. After the intensity modulation with the actual RF signal, the transmission through the fibre-optic path takes place. Finally, at the fibre end the PD receiver extracts the RF signal by direct detection (DD).

\section{OPTO-ELECTRONIC OSCILLATOR}

A high-performance oscillator with a high spectral purity is a key element in every wireless communication system. For RF, microwave and $\mathrm{mmW}$ signal generation, different approaches are used, such as quartz crystal oscillators, dielectric resonators, sapphire resonators or air-dielectric resonators. Unfortunately, in all these implementations the phase noise of the oscillator increases with the frequency. A device that is not subject to this restrictive law of physics is the OEO, which was introduced in the early 1990s [13]. Since then, the device's key properties have been continuously improved and the design issues solved [14], [15], [16], [17].

A schematic diagram of a single-loop OEO is shown in Fig. 1. The main component of the OEO is a low-loss optical fibre, which acts as very long delay line. The biggest advantage of an OEO is that the resonator quality factor (Q) is proportional to the product of the time delay introduced by the fibre and the oscillator's frequency. Since the loss of the optical fibre is determined by the LD's wavelength, it stays constant for practically any generated signal frequency. As a result of this, Q increases if the oscillation frequency is increasing. In other words, if the length of the OEO fibre-optic link increases, the phase noise of the OEO will decrease [14].

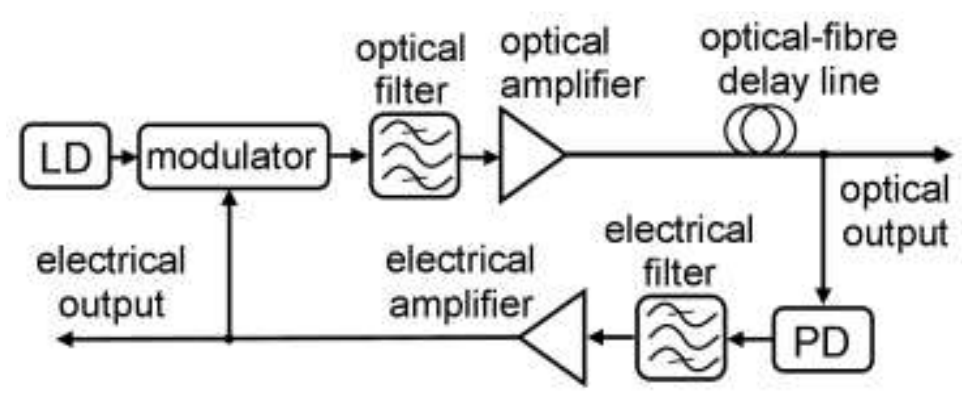

Figure 1. Optical, electrical and optoelectronic components of a single-loop opto-electronic oscillator (OEO).

A single-loop OEO consists of an optical and an electronic section. Each of these parts has its own signal output, i.e., optical and electrical. In our proposal for the distribution of a low-phase-noise oscillator signal, we would like to use the optical signal output and deliver it via an optical fibre to the base-station, where it is converted to an electrical form and used.

\section{SYSTEM APPROACH}

Nowadays, a lot of $2 \mathrm{G}, 3 \mathrm{G}$ and $4 \mathrm{G}$ base-stations are already connected to a central office via fibre-optic links with the purpose of bringing a broadband signal to the end user's mobile devices. Essentially, such mobile networks are a combination of wireline and wireless transmission. In the base-station the conversion between the optical and radio-frequency signal occurs. Over large distances the transmission from the central office to the base-stations is achieved via fibre optics, while over smaller distances, within the cell area, transmission from the base-station to the user's mobile devices is achieved by wireless radio interfaces.

The common principle, which has been in use for a number of years and can be seen in currently available $2 \mathrm{G}, 3 \mathrm{G}$ and $4 \mathrm{G}$ commercial cellular networks, is illustrated in Figure 2. Optical fibre is used as an effective substitute for a coaxial cable or a twisted pair. Through a fibre-optic line, comprising an emitting LD and PD, the transmission is achieved with PDH, SDH or ATM, which are currently being replaced by IP. With such a solution the transition from wired to wireless transmission requires a signal conversion at each base-station. On the transmitter side of the base-station, the frequency mixer and a reference low-phase-noise local oscillator (LO) are used to up-convert the baseband signal into the modulated carrier needed for the wireless transmission. Similarly, the frequency mixer and the low-phase-noise LO are used for the down-conversion of the incoming signal on the receiver side of the base-station. 


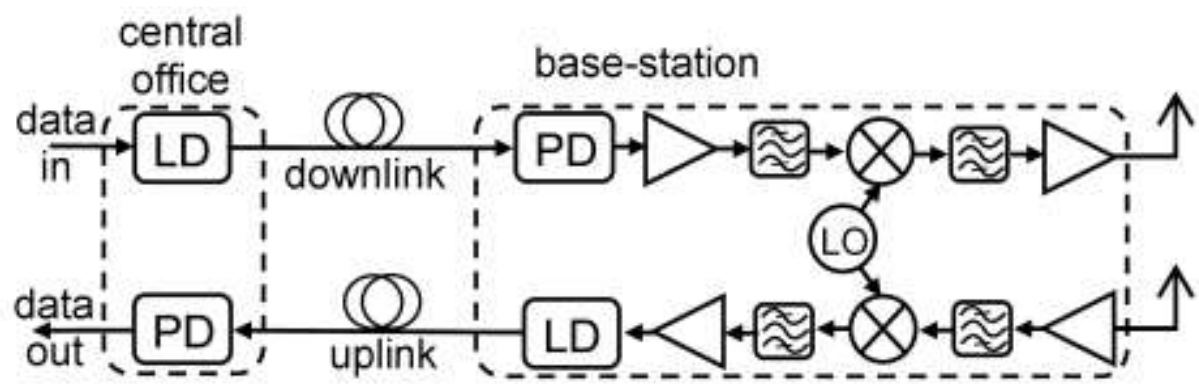

Figure 2. Transmission over optical fibre in the baseband and signal processing at the base-station.

If different frequencies are used for signal transmission and reception, the base-station is usually equipped with two separate antennas. It is also possible to use a single antenna simultaneously for the signal transmission and reception by making the receiver output fully reflective when powered off [18] or by implementing a magnetic circulator or even with an integrated chip circulator [19], which routes the signals from the radio transmitter to the antenna and from the antenna to the radio receiver. This singleantenna solution is especially useful for half-duplex communication, but in in-band full-duplex communications a large amount of transmitted signal can leak to the radio receiver in the case of antenna impedance mismatching. Since the transmitted signal power is much higher than the received signal power, even a small leak can completely destroy the receiving signal if a canceler circuit [19] is not used.

High-capacity broadband wireless systems that are based on orthogonal frequency-division multiplexing (OFDM) or the latest non-OFDM [5] require a low-phase-noise RF signal source with a high stability located in the temperature-regulated environment of the base-station. A sufficiently accurate, oven-stabilised crystal oscillator increases the complexity of the base-station and is a very expensive solution. When the number of cells starts to increase with the appearance of $5 \mathrm{G}$, such mobile networks will become uneconomical due to the large number of base-stations that are applied with today's technology. For this reason a new technology to overcome the problem is required.

One of the possible solutions is to generate a microwave signal by optical heterodyning [20], wherein the LDs are located in a central office and the light from them is transmitted over optical fibre to the base-station where those two optical waves of different wavelengths are beaten at the PD that is located in the base-station [21], as shown in Fig. 3. The RF signal generation through optical photo-mixing eliminates the need for any frequency stabilisation of the local oscillator in the base-station.

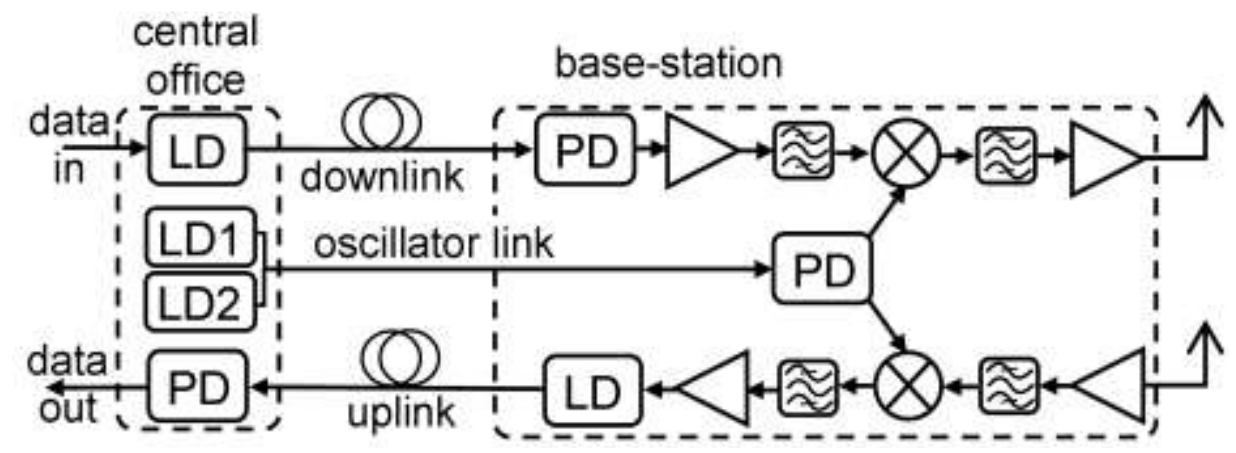

Figure 3. Transmission of two optical signals from a central office and optical mixing to generate a microwave or mmW signal on the PD in the base-station.

In this paper we are proposing to have a low-phase-noise OEO at the central office instead of two LDs. Figure 4 shows how the single-loop OEO can be implemented in a RoF system in order to simplify the network's base-stations. The low-phase-noise signal from the optical output of the OEO is transferred from the central office to the base-station by separate single-mode optical fibres.

Furthermore, we are proposing to use only one OEO in the central office and distribute its signal to many base-stations by using RoF technology and a passive optical network. If the low-phase-noise oscillator signal is transmitted from the central office over a passive optical network infrastructure, the number of oscillators in the system is reduced. We believe that in a practical network with a large number of terminal base-stations and one central office, the above type of proposed RoF transmission brings a significant economic advantage. 


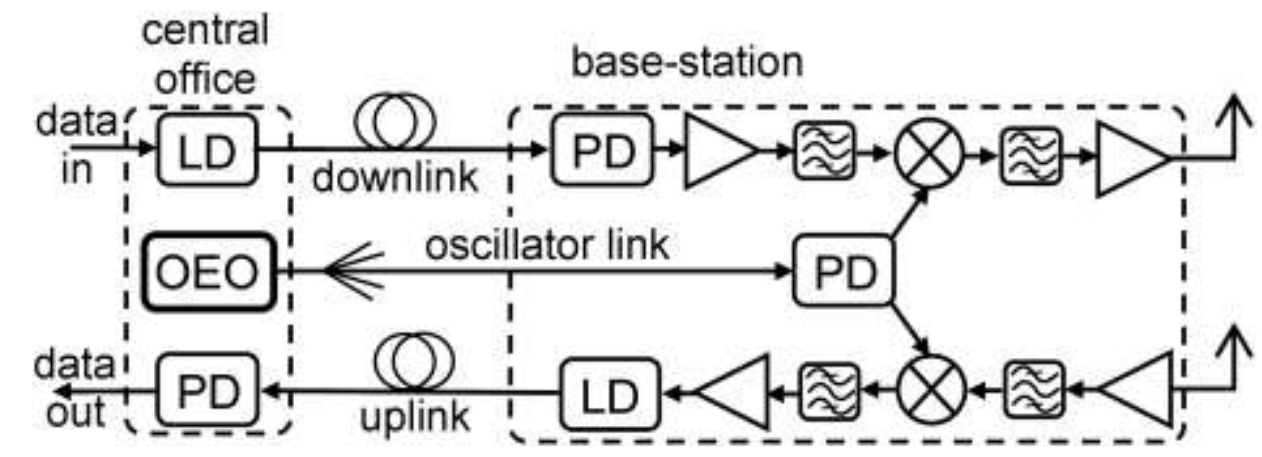

Figure 4. RoF transmission of low-phase-noise oscillator signal over optical fibre and optical detection at the base-station.

At the remote base-station, the RF signal is detected by the PD, creating a RF signal suitable for the frequency conversion of the data signal in the mixer. If we wish to create an RF signal through photo-detection, the PD needs a sufficient bandwidth for the relevant frequency area.

A solution that uses separate optical lines for the data signal and for the mixing signal is shown in Figure 4 . Of course, it is possible to join the signals from all three fibre-optic lines in a single optical fibre using wavelength-division multiplexing (WDM) technology. It should be noted that there are certain disadvantages of distributing the oscillator signal from the central office. The main one is that the optical signal that carries the oscillator signal at the output of the OEO is intensity modulated. It is well known that amplitude modulation is highly sensitive to chromatic dispersion of the optical fibre, which accumulates with the length of the fibre [22]. As the modulated optical signal is transmitted via a fibre-optic link, the chromatic dispersion causes a different phase shift on each of the spectral components (the carrier and double sidebands), as shown in Figure 5.

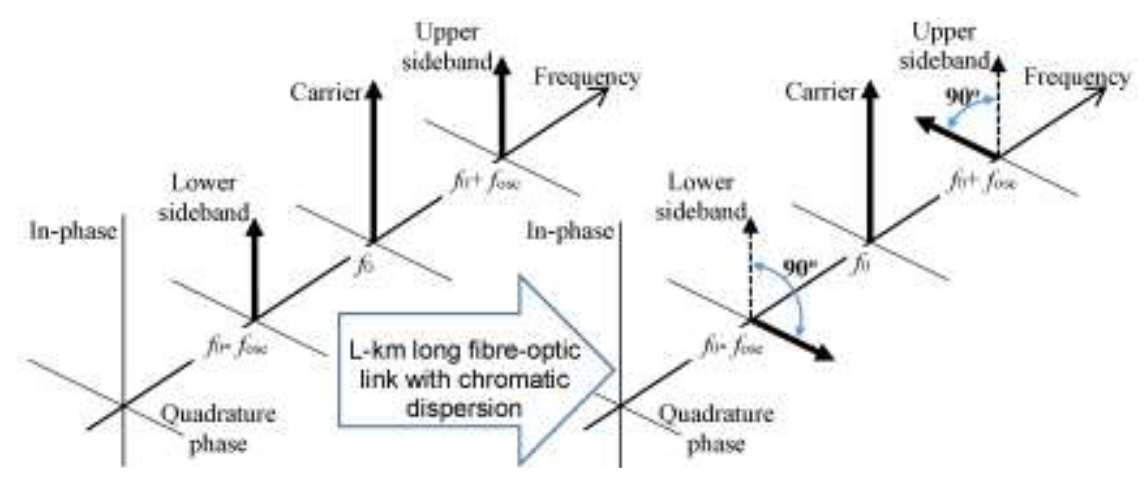

Figure 5. Spectrum of intensity-modulated signal at the fibre input and after L-km of fibre. Chromatic dispersion changes the phase relationship between the optical carrier and upper and lower sidebands.

The phase shift of each spectral component depends on the fibre length, the oscillator frequency and the dispersion coefficient. Consequently, the power of the detected signal is length dependent [21].

$$
P_{\text {osc }}\left(L, f_{\text {osc }}\right) \propto 10 \log \left(\cos \left(\frac{\pi \cdot L \cdot D}{c_{0}}\left(\lambda_{0} \cdot f_{\text {osc }}\right)^{2}\right)\right)
$$

where $L$ is the fibre length, $c_{0}$ is the speed of light in free space, $D$ is the fibre-dispersion coefficient, $\lambda_{0}$ is the optical carrier wavelength, and $f_{\text {osc }}$ is the oscillator frequency. In the case when the phase delay between the sidebands at the end of the fibre line is $180^{\circ}$, the destructive mixing on the PD will negate the entire oscillator signal, as shown in Figure 6. 


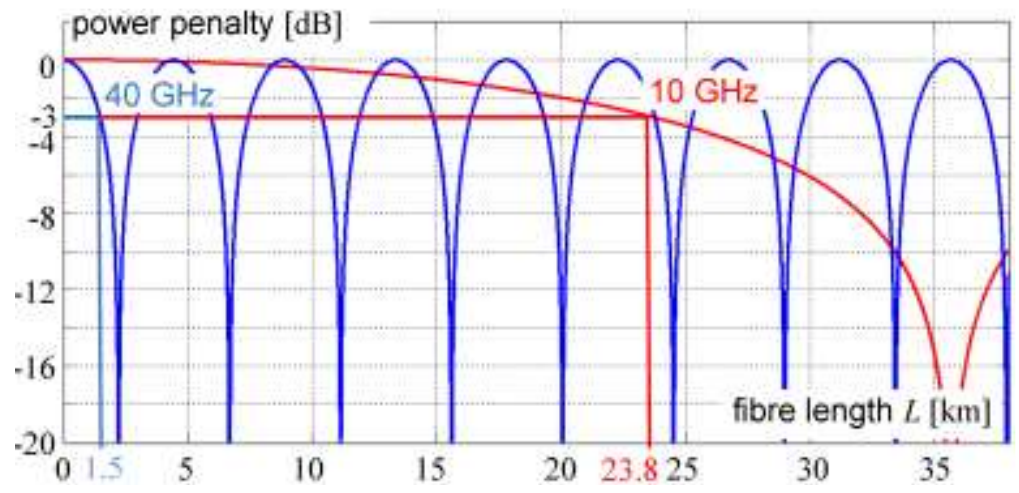

Figure 6. Power penalty of the oscillator signal depend on the fibre length.

For example, when a $10-\mathrm{GHz}$ oscillation frequency is transmitted over the fibre-optic link at $1550 \mathrm{~nm}$, where the standard single-mode fibre that has a $17.5 \mathrm{ps} /(\mathrm{nm} . \mathrm{km})$ dispersion, a $3-\mathrm{dB}$ degradation in the oscillator signal occurs at a distance of $23.8 \mathrm{~km}$. At a $40-\mathrm{GHz}$ oscillator frequency a 3-dB degradation occurs after just $1.5 \mathrm{~km}$. It seems that the transmission of the oscillator signal by an intensity-modulated optical signal is only applicable for frequencies up to $10 \mathrm{GHz}$. For higher oscillator frequencies the limiting dispersion occurrence can be reduced by using an OEO, where a single sideband modulation [23] with an optical filtration or specially controlled MZM is used.

Besides chromatic dispersion, the polarization mode dispersion (PMD) of a fibre-optic link can also cause a degradation in the quality of the transmitted oscillator signal. Consequently, a low-PMD fibre-optic link is required. However, attention must be paid to the Rayleigh scattering of the fibre-optic link and its contribution to the phase noise.

\section{CONCLUSION}

The information capacity for $5 \mathrm{G}$ mobile networks continues to increase, making it advantageous to use mmW frequency bands and smaller cells. High frequencies require oscillators with a high stability and a low phase noise. In this paper we introduce a RoF technology, which is known as being very suitable for the transmission of RF signals, and a stable, low-phase-noise OEO. This paper attempts to bring those two technologies closer by highlighting the specific advantage of minimising mobile communication system costs with the distribution of the oscillator signal from one OEO to many base-stations using RoF. For the distribution of the oscillator signal we propose a passive fibre-optic network with a tree topology. As a final contribution, the paper also outlined a few phenomena that may influence the transmission of an oscillator signal to the base-station. We lay out the possible system limitations, such as chromatic dispersion, PMD and scattering, which need to be addressed in further research. An IM-DD RoF system is susceptible to fibre-optic link chromatic dispersion, which induces the length- and frequency-dependent suppression of the RF power. In future research we plan to study the performance of a distributed oscillator system with an OEO as well as experimentally evaluating the system's limitations. We are also interested in a multi-frequency delivery system using RoF technology. Last but not least, we plan to continue to research novel stabilisation techniques in combination with side-modes suppression for an OEO.

\section{ACKNOWLEDGMENT}

The authors would like to express their gratitude to the company InLambda BDT d.o.o. for the research equipment and devices. The work presented in this article was created in collaboration with ARRS Algorithms and optimisation procedures in telecommunications programme and FiWIN5G Innovative Training Network, which has received funding from the European Union's Horizon 2020 Research and Innovation Programme 2014-2018 under the Marie Sklodowska-Curie grant agreement No.642355.

\section{REFERENCES}

[1] P. K. Agyapong, et al., "Design considerations for a 5G network architecture," IEEE Comm. Mag., vol. 52, no. 11, pp. 6575, Nov. 2014.

[2] W. Hong, K. H. Baek, Y. Lee, Y. Kim, S. T. Ko, "Study and prototyping of practically large-scale mmWave antenna systems for $5 \mathrm{G}$ cellular devices," IEEE Commun. Mag., vol. 52, no. 9, pp. 63-69, Sept. 2014.

[3] W. Afrić and S. Z. Pilinsky, "UMTS LTE downlink cell size calculation," Proc. ELMAR, 2012 Zadar, 2012, pp. 105-108.

[4] J. Vihriala, N. Ermolova, E. Lahetkangas, O. Tirkkonen and K. Pajukoski, "On the Waveforms for 5G Mobile Broadband Communications," 2015 IEEE 81st Vehicular Technology Conf. (VTC Spring), Glasgow, 2015, pp. 1-5.

[5] R. G. Clegg, S. Isam, I. Kanaras and I. Darwazeh, "A practical system for improved efficiency in frequency division multiplexed wireless networks," IET Commun., vol. 6, no. 4, pp. 449-457, March 62012. 
[6] P. Robertson and S. Kaiser, "Analysis of the effects of phase-noise in orthogonal frequency division multiplex (OFDM) systems," IEEE Int. Conf. on Commun., ICC '95, Seattle, WA, 1995, pp. 1652-1657 vol. 3.

[7] Z. Pi and F. Khan, "An introduction to millimeter-wave mobile broadband systems," IEEE Commun. Mag., vol. 49, no. 6, pp. 101-107, June 2011.

[8] Z. Pi, J. Choi and R. Heath, "Millimeter-wave gigabit broadband evolution toward 5G: fixed access and backhaul," IEEE Commun. Mag., vol. 54, no. 4, pp. 138-144, April 2016.

[9] M. Vidmar, "Optical-fiber communications: Components and Systems," Inform. MIDEM, vol. 31, no. 4, pp. $246-251,2001$.

[10] B. Batagelj, V. Janyani, S. Tomažič, "Research challenges in optical communications towards 2020 and beyond," Inform. MIDEM, vol. 44, no. 3, pp. 177-184, 2014.

[11] Nathan J. Gomes, et al., "Radio over fiber transport for the support of wireless broadband services," J. Opt. Netw., vol.8, no.2, pp.156-178, Feb. 2009.

[12] John E. Mitchell: Radio-over-Fiber (RoF) Networks, in Broadband Access Networks: Technologies and Deployments, Springer, 2009.

[13] R.T. Logan, L. Maleki, M. Shadaram, "Stabilization of oscillator phase using a fiber-optic delay-line", in Proc. 45th Annu. Symp. on Frequency Control, pp. 508-512, May 1991.

[14] B. Batagelj, L. Bogataj and M. Vidmar, "Key properties and design issues for an opto-electronic oscillator," 17th Int. Conf. on Transparent Optical Networks (ICTON), Budapest, 2015, pp. 1-4.

[15] L. Bogataj, M. Vidmar and B. Batagelj, "A Feedback Control Loop for Frequency Stabilization in an Opto-Electronic Oscillator," J. Lightwave Technol., vol. 32, no. 20, pp. 3690-3694, Oct.15, 2014.

[16] L. Bogataj, M. Vidmar and B. Batagelj, "Improving the Side-mode Suppression Ratio and Reducing the Frequency Drift in an Opto-Electronic Oscillator With a Feedback Control Loop and Additional Phase Modulation," J. Lightwave Technol., vol. 34, no. 3, pp. 885-890, Feb., 2016.

[17] L. Bogataj, M. Vidmar and B. Batagelj, "Opto-Electronic Oscillator With Quality Multiplier," IEEE T. Microw. Theory, vol. 64, no. 2, pp. 663-668, Feb. 2016.

[18] M. Vidmar, "Use transmitting power FETs for antenna switching," in Microwaves \& RF, vol. 39, no. 7, pp. 81-86, 2000.

[19] A. Sabharwal, P. Schniter, D. Guo, D. W. Bliss, S. Rangarajan and R. Wichman, "In-Band Full-Duplex Wireless: Challenges and Opportunities," IEEE J. Sel. Area. Comm., vol. 32, no. 9, pp. 1637-1652, Sept. 2014.

[20] J. Yao, "Microwave Photonics," J. Lightwave Technol., vol. 27, no. 3, pp. 314-335, Feb.1, 2009.

[21] Bostjan Batagelj, et al.: Convergence of fixed and mobile networks by radio over fibre technology, Inform. MIDEM, vol. 41, no. 2, pp. 144-149, June 2011

[22] H. Schmuck, "Comparison of optical millimetre-wave system concepts with regard to chromatic dispersion," in Electron. Lett., vol. 31, no. 21, pp. 1848-1849, 12 Oct 1995.

[23] G. H. Smith, D. Novak and Z. Ahmed, "Overcoming chromatic-dispersion effects in fiber-wireless systems incorporating external modulators," IEEE Trans. Microw. Theory Techn., vol. 45, no. 8, pp. 1410-1415, Aug 1997. 